\title{
Poliarterite nodosa due to anti elastase antibody
}

\author{
Caterina Defendenti', Aldo Cereda², Mariella Saudelli' , Maurizio Picca² \\ ' Laboratorio di Analisi, Ospedale Fatebenefratelli, Milano \\ ${ }^{2}$ Unità di Medicina Interna, Ospedale Macedonio Melloni, Milano
}

Key words: ANCA, Elastase, Polyarteritis nodosa, Vasculitis, Antineutrophil cytoplasmic autoantibodies

\section{Un caso di poliarterite nodosa anti-elastasi e ANA positiva}

\section{SUMMARY}

The Authors related one case of polyarteritis nodosa occurred to a men forty eight years old. The clinical was characterized by mesenteric and femoral arteries occlusion and chronic cutaneous ulcers to legs. There were bioptical aspects of systemic vasculitis with necrotizing inflammation and a paucity of immune deposit. It was effective oral cyclophosphamide plus steroids. This disease was closely associated with antibodies anti elastase (HLE). The patient had not a history of cocaine abuse or LES disease but the nucleolar pattern ANA was positive >1:640 (anti-nDNA negative). Similar case ANA positive associated with the anti-elastase antibodies, was described by Nassberger (Lancet 1989) for 6/I04 patients with LES, anti-nDNA negative. The patient with the highest anti-elastase concentration subsequentely died after very rapid development of severe brain and kidney involvement.

Received February I, 2008

Accepted February 18, 2008

\section{INTRODUZIONE}

Le vasculiti sistemiche comprendono uno spettro di sindromi cliniche caratterizzate da infiammazione e necrosi dei vasi sanguigni (7). Gli anticorpi anti citoplasma dei neutrofili sono il marker sierologico di un significativo gruppo di pazienti con queste patologie. In particolare sono stati ben individuati anticorpi contro gli antigeni maggiori PR3 e MPO rispettivamente cANCA (fluorescenza citoplasmatica) e pANCA (fluorescenza perinucleare). Essi sono riconosciuti come causa delle più importanti vasculiti sistemiche come la Granulomatosi di Wegener (PR3 positiva) o la sindrome di Churg-Strauss (MPO positiva).

I neutrofili umani però contengono altri tre tipi di granuli con diversi costituenti proteici: i granuli azzurrofili che oltre a PR3 e MPO contengono BPI (bactericidal permeability increasing protein), elastasi, catepsina $\mathrm{G}$ e azurocidina, i granuli secondari con lactoferrina e lisozima e i granuli terziari contenenti gelatinasi. Ciascuno di questi granuli è un target potenziale per una reazione autoimmune. Non esiste in letteratura una chiara associazione tra anticorpi diretti contro questi antigeni "minori" e le vasculiti sistemiche. Essi sono stati chiaramente identificati solo in forme localizzate, come in patologie infiammatorie intestinali mal responsive al trattamento chirurgico. Nelle colangiti sclerosanti si riscontra positività nel $100 \%$ dei casi per pANNA (antineutrophil nuclear antibodies) il cui pattern antigenico non è ancora stato identificato.

Nel caso descritto sono presenti nel siero del paziente elevati livelli di autoanticorpi anti elastasi con metodo ELISA (index 8.8); in IFI essi si rivelano con marcata fluorescenza a distribuzione grossolana citoplasmatica sui vetrini fissati in formalina. Sui vetrini fissati in etanolo invece la distribuzione è perinucleare con addensamento tanto blando intorno al nucleo da sembrare una distribuzione citoplasmatica. In effetti l'elastasi ha una stretta omologia con PR3 e qualche volta simula un aspetto cANCA in immunofluorescenza. In combinazione con PR3, inoltre stimola il fattore tissutale endoteliale e può indurre infiammazione vascolare (3).

Weisner, et al. (10) sostengono che gli autoanticorpi anti elastasi sono frequenti nelle lesioni del setto indotte da cocaina e che la presenza di anticorpi anti elastasi può discriminare questa entità clinica dalla Granulomatosi di Wegener. In un lavoro di Talor (7) che prende in considerazione un ampio gruppo di soggetti, sono stati riscontrati pochissimi casi di positività anti elastasi, nessuno dei quali associato all'abuso di cocaina. Dei casi segnalati, solo ad un paziente era stata diagnosticata Granulomatosi di Wegener. Tutti gli altri erano definiti come "other diagnosis". La rarità 
della malattia rende ragione delle difficoltà di classificazione.

Interessante è però uno studio del 1989 di Nassberger, pubblicato da Lancet, (5) che arruola un ampio gruppo di pazienti con malattie reumatiche e che rileva anticorpi anti elastasi solo in 6 pazienti con diagnosi di LES, nDNA negativi, con concomitante positività ANA ad alti livelli. La storia evolutiva dei pazienti selezionati dall'autore è stata caratterizzata da un prevalente interessamento del sistema nervoso centrale, principale causa di morte. Nassberger individua così negli anticorpi anti elastasi un marker di coinvolgimento neurologico in pazienti con LES, ANA positivo e anti-nDNA negativo.

Il caso clinico che verrà descritto riguarda quindi un soggetto di sesso maschile che presenta un quadro sierologico infrequente e sovrapponibile a quanto riporta nello studio pubblicato da Lancet con diagnosi clinica e istologica di poliarterite nodosa.

La poliarterite nodosa (PAN) è una vasculite necrotizzante delle arterie di medio e di piccolo calibro. L'età media d'insorgenza è di 45 aa e colpisce prevalentemente i maschi con un rapporto 2.5:1.

Le lesioni sono segmentarie e hanno una predilezione per i punti di ramificazione dei vasi che sono interessati a tutto spessore con facile evoluzione aneurismatica.

È presente un infiltrato cellulare rappresentato da granulociti neutrofili e macrofagi e caratteristicamente sono presenti zone di necrosi fibrinoide. La parete vasale può restringersi e il lume può occludersi per la formazione di un trombo con ricorrenti episodi ischemici. Alternativamente possono formarsi piccoli aneurismi che, quando interessano arterie superficiali, possono essere palpati; la loro presenza denomina la malattia. Uno degli aspetti laboratoristici costanti è la leucocitosi neutrofila, a volte spiccata.

\section{CASO CLINICO}

Un uomo di 48 anni è stato ricoverato presso l'U.O. di Medicina Interna dell'Ospedale Macedonio Melloni per la comparsa, nei 2 mesi precedenti il ricovero, di febbre e di ulcere torpide agli arti inferiori, già trattate con terapia antibiotica imprecisata senza beneficio.

L'esame obiettivo evidenziava assenza bilaterale dei polsi arteriosi tibiale e popliteo, ulcere torpide a fondo sanioso delle gambe e dei piedi, ed ipoacusia bilaterale. Nella storia clinica del paziente veniva segnalato tabagismo ed un ricovero ospedaliero all'età di 42 anni per sordità bilaterale ad insorgenza acuta (del quale non veniva allegata documentazione). Il paziente riferiva inoltre una

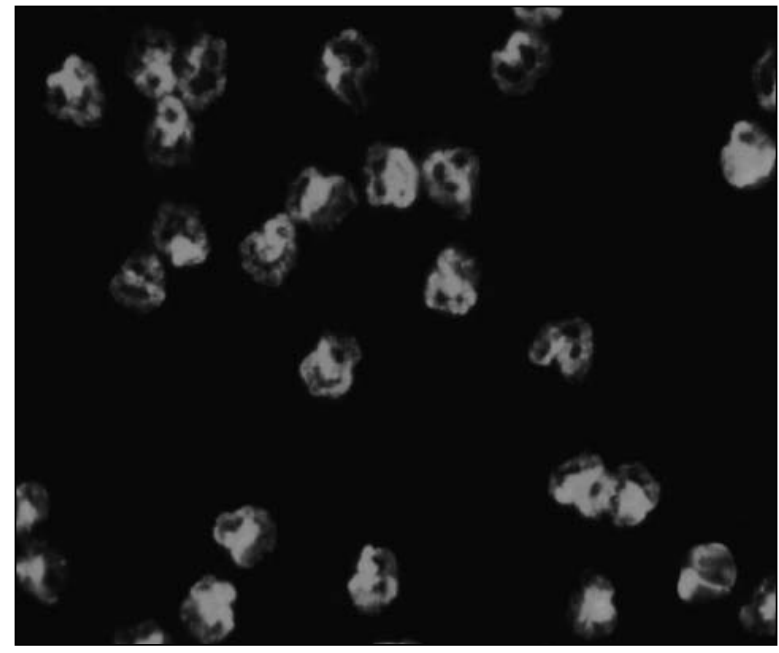

Figura I. Ab anti elastasi su vetrino IFI fissato con metanolo.

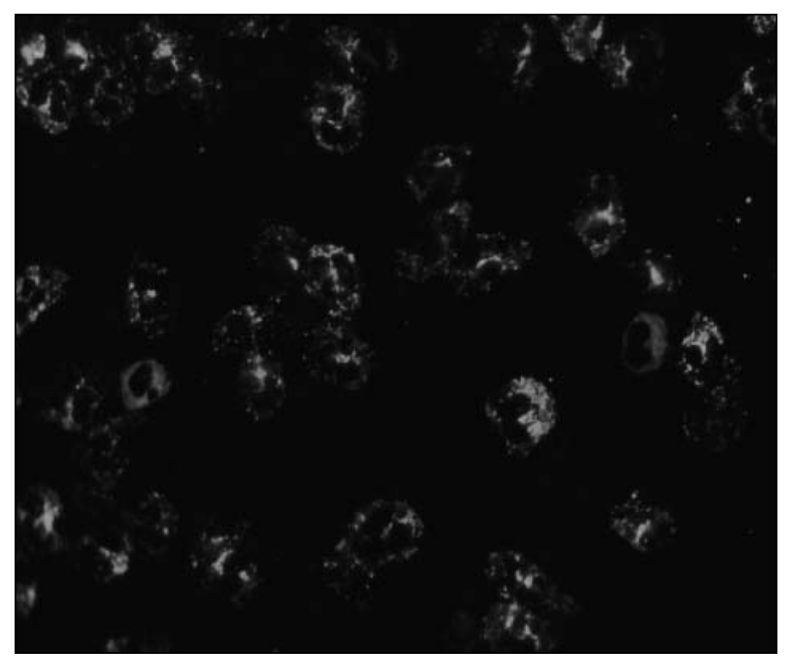

Figura II. Ab anti elastasi su vetrino IFI fissato con formalina.

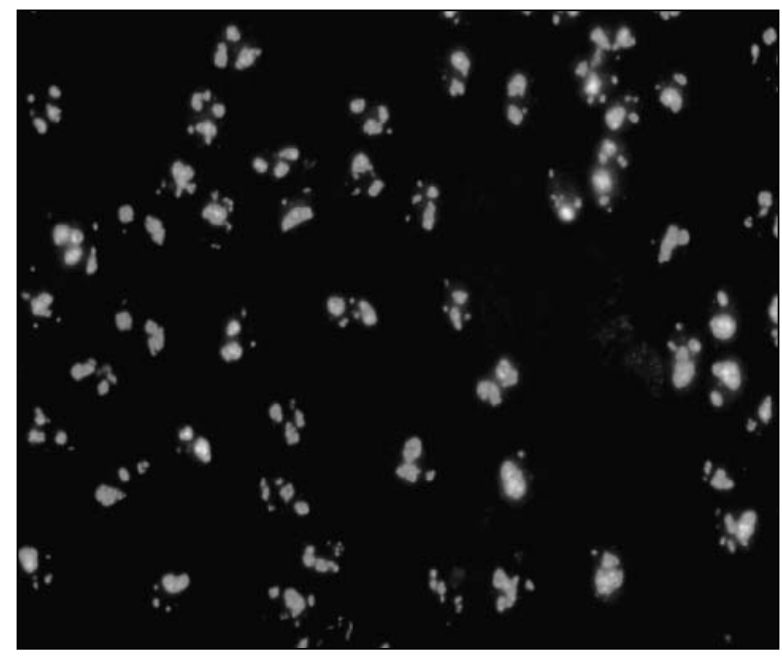

Figura III. IFI su cellule HP2; ANA positivo I:640 con pattern nucleolare.

puntura di zecca avvenuta circa 4 mesi prima del ricovero e non trattata. L'esame arteriografico dimostrava occlusione completa della mesenterica superiore con circolo collaterale attraverso l'arca- 
ta di Riolano, stenosi serrata $1 \mathrm{~cm}$. dopo l'origine della femorale superficiale sinistra ed irregolarità parietali con placca ulcerata nel tratto infragenicolato della poplitea, occlusione completa della femorale superficiale $\mathrm{dx}$ all'origine con rivascolarizzazione distale tramite circoli collaterali della femorale profonda. La TAC del torace dimostrava estesa distrofia bollosa bilaterale ai campi polmonari medio e superiore, mentre alla TAC dell'encefalo era presente ipodensità diffusa della sostanza bianca. Gli esami ematochimici evidenziavano incremento della PCR (35), leucocitosi neutrofila (GB: 17.100; N: 78\%), positività degli ANA (1:640, pattern nucleolare omogeneo) e positività per pANCA e Ab anti-elastasi. Una biopsia delle lesioni cutanee dimostrava frammenti cutanei con ulcerazioni diffuse, necrosi e densi infiltrati flogistici estesi sino all'ipoderma costituiti da granulociti neutrofili, linfociti, istiociti e rari eosinofili coesistenti con vasculite leucocitoelastica ed aspetti di endoarterite (quadro istologico coerente con la diagnosi di vasculite, suggestiva per una forma di panarterite nodosa).

Il paziente veniva posto in terapia con prednisone $60 \mathrm{mg} /$ die e ciclofosfamide $100 \mathrm{mg} /$ die con rapida risoluzione dello stato febbrile e progressivo miglioramento delle lesioni cutanee degli arti inferiori. Ad una visita di follow-up eseguita 3 mesi dopo la dimissione si osservava regressione completa delle lesioni cutanee e normalizzazione degli indici di flogosi.

\section{DISCUSSIONE}

Fino ad un decennio fa la granulomatosi di Wegener e la Sindrome di Churg-Strauss rientravano tra le patologie ad etiologia sconosciuta. Attualmente il rilievo di ANCA specifici costituisce uno dei parametri maggiori per la loro diagnosi.

$\mathrm{Nel}$ caso in questione si configura un aspetto particolare, già segnalato da Nassberger, in cui, dal punto di vista sierologico coesistono positività ANA molto intensa (nDNA negativa) ed anticorpi anti elastasi. Queste caratteristiche erano state rilevate dall'autore su 6 di 104 pazienti per i quali era stata fatta diagnosi di LES, tutti positivi per ANA ed anti-elastasi, ma negativi per anticorpi anti-nDNA. L'articolo riporta che i pazienti con più alta concentrazione di anticorpi anti elastasi sono successivamente deceduti dopo un rapidissimo coinvolgimento del cervello e del rene. L'autore quindi propone che l'anti elastasi possa essere un marker di coinvolgimento neurologico in alcuni pazienti con LES, in assenza di anamnesi per malattia indotta da droghe.

Gli autoanticorpi diretti contro "antigeni minori" dei neutrofili necessitano di attenzione in quanto possono essere marker di sindromi vasculitiche sistemiche. Si tratta di un campo non ancora ben esplorato e spesso di difficile inquadramento ma che può configurare sindromi di estrema gravità $\mathrm{e}$ rapidamente evolutive. Una diagnosi ed un trattamento adeguato migliorano drammaticamente la prognosi.

\section{BIBLIOGRAFIA}

1. Gang X, Ming-hui Z, et al. Detection rate and antigenic specificities of antineutrophil cytoplasmic antibodies in Chinese patients with clinically suspected vasculitis Clinical and Diagnostic Laboratory Immunology. May 2004; 559-62.

2. Gross W, Csernok E. Antineutrophil cytoplasmic autoantibodies, autoantigenes, and systemic vasculitis. APMIS 1995; 103: 81-97.

3. Haubitz M, Gerlach M, et al. Endothelial tissue factor stimulation by proteinase 3 and elastase. Clin Exp Immunol 2001; 126: 584-8.

4. Kamesh L, Harper L, Savage CO. ANCA-positive vasculitis. J Am Soc Nephrol 2002; 13: 1953-60.

5. Nassberger L, Jonasson H, et al. Circulating anti-elastase in systemic lupus erythematosus. Lancet 1989; 1: 509.

6. Savige J, Gillis D, Benson E, et al. International Consensus Statement on Testing and Reporting of Antineutrophil Citoplasmic Antibodies (ANCA). Am J Clin Pathol 1999; 111: 507-13.

7. Talor MV, Stone JH, et al. Antibodies to selected minor target antigens in patients with anti-neutrophil cytoplasmic antibodies (ANCA). Clinical and Experimental Immunology 2007; 150: 42-8.

8. Van der Woulde F, Rassmussen N, Lobatto S, et al. Autoantibodies against neutrophil and monocytes: tool for diagnosis and marker of disease activity in Wagener's granulomatosis. Lancet 1985; i: 425-9.

9. Watts R, Scott G. Classification and epidemiology of the vasculitides. Baillieres Clin Rheumatol 1997; 11: 191-217.

10. Weisner O, Russell KA, et al. Antineutrophil cytoplasmic antibodies reacting with human neutrophil elastase as a diagnostic marker for cocaine-induced midline destructive lesions but not autoimmune vasculitis. Arthritis Rheum 2004; 50: 2954-65. 